\title{
High-Level Resistance to Ciprofloxacin and Rising MIC to Ceftriaxone and Azithromycin among Enteric Fever Isolates from a Tertiary Care Center, Puducherry, India
}

\author{
K. Sandhya Bhat, S. Anandhalakshmi*, Desdemona Rasitha, \\ Shreyas Venkataraman and Reba Kanungo
}

Department of Microbiology, Pondicherry Institute of Medical Sciences, Puducherry, India

*Corresponding author

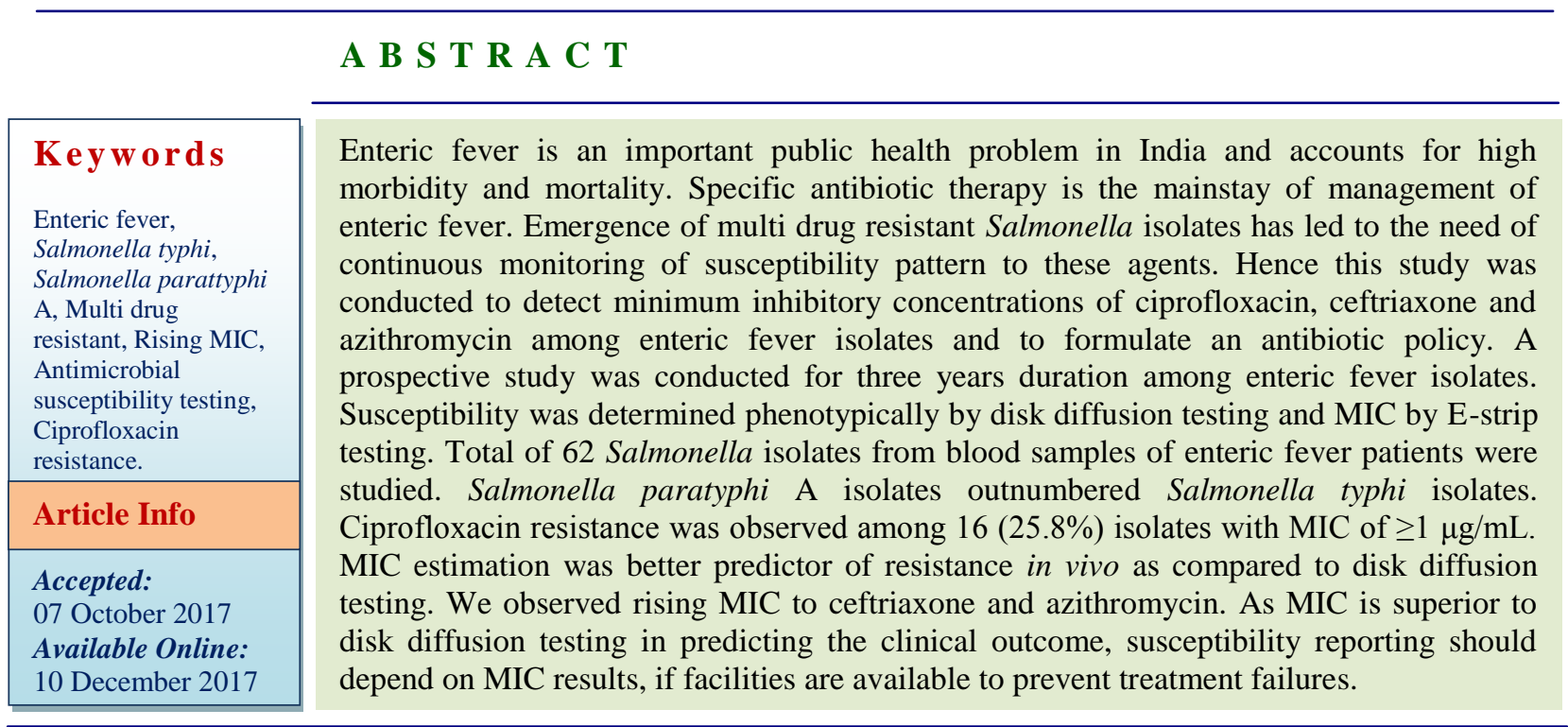

\section{Introduction}

Enteric fever is an important public health problem in India with Salmonella enterica var typhi (S. typhi) and Salmonella enterica var paratyphi A (S. paratyphi A) being the leading causative agents (Singhal et al., 2014). These human restricted pathogens pose great threat in regions with poor standards of hygiene and sanitation and they account for high morbidity and mortality both among pediatric and adult populations (Adabara et al., 2012). Specific antibiotic therapy is the mainstay of management of enteric fever; if left untreated then mortality can be as high as $30 \%$, and with appropriate treatment mortality rate reduces to as low as $0.5 \%$ (Menezes et al., 2012).

In the past few decades, there have been changes documented by various researchers across India, in both epidemiology and drug resistance pattern of enteric fever isolates. Firstly, there has been an increasing trend of S. paratyphi A over the last decade in India (Gupta et al., 2009; Singhal et al., 2014). 
Secondly, there has been a noted reemergence of susceptibility to first-line antibiotics, such as ampicillin, co-trimoxazole and chloramphenicol; which were earlier used for the treatment of enteric fever cases before the era of multi drug resistant (MDR) Salmonella (Choudhary et al., 2013; Gupta et al., 2009; Raveendran et al., 2008). Thirdly, emergence of reduced susceptibility to ciprofloxacin and slow rise of minimum inhibitory concentrations to $3^{\text {rd }}$ generation cephalosporins and azithromycin, which are used as current front line drugs in the treatment of enteric fever (Capoor et al., 2007; Garget al., 2013; Srirangaraj et al., 2014). However, recent reports of decreased susceptibility to these agents have led to the prospect of re-emergence of untreatable enteric fever and an increasing global burden and hence, continual monitoring of drug resistance is imperative (Choudhary et al., 2013; Kawser et al., 2013).

Considering wide variation in the susceptibility pattern of various strains of Salmonella among different geographical areas, with high endemicity, it is mandatory to test antimicrobial susceptibility before instituting definite therapy (Menezes et al., 2012; Singhal et al., 2014). Therefore, this study was undertaken to detect antimicrobial susceptibility profile and minimum inhibitory concentrations of ciprofloxacin, ceftriaxone and azithromycin among enteric fever isolates and thereby formulating an antibiotic policy for management of drug resistant enteric fever cases in our tertiary care hospital.

\section{Materials and Methods}

A prospective study was carried out after obtaining ethics clearance from Institute Ethics Committee (RC No- RC/14/104), at department of Microbiology, Pondicherry Institute of Medical Sciences, Pondicherry for a duration of three years from June 2013 to
May 2016. A total of sixty two clinical isolates of Salmonella from clinically suspected enteric fever patients were included. Identification of salmonellae isolates was performed using standard biochemical tests and isolates were confirmed by serotyping with specific Salmonella polyvalent antisera $\mathrm{O}$ and with $\mathrm{O} 9$ or $\mathrm{O} 2$ antisera (Koneman's Color Atlas and Textbook of Diagnostic, 2007).

Antimicrobial susceptibility testing was performed by using Kirby- Bauer disk diffusion method by using0.5 McFarland bacterial suspension on Mueller-Hinton agar. Commercially available disks (Hi-media Laboratories, Mumbai, India) of ampicillin (10 $\mu \mathrm{g})$, chloramphenicol $(30 \mu \mathrm{g})$, co- trimoxazole $\quad(1.25 / 23.75 \quad \mu \mathrm{g})$, ciprofloxacin $(5 \mu \mathrm{g})$, ceftriaxone $(30 \mu \mathrm{g})$ and azithromycin $(15 \mu \mathrm{g})$ were tested.

Minimum inhibitory concentrations (MICs) of ciprofloxacin, ceftriaxone and azithromycin for all Salmonella isolates were determined by Epsilometer strip test method (HiMedia, Mumbai, India).

The antimicrobial agent concentration, at which edge of the inhibition ellipse intersects the side of the E-strip, was taken as MIC value. Escherichia coli ATCC 25922 was used as control for both the disc diffusion and MIC testing.

Results of disk diffusion as well as MIC breakpoints were interpreted as per CLSI2015 guidelines except for azithromycin (as its interpretation is not given in this document). As CLSI 2017 gives interpretative criteria for azithromycin disc diffusion testing and azithromycin MIC breakpoints only for $S$. typhi isolates, same was used for interpretation of azithromycin results for $S$. typhi isolates and also for $S$. paratyphi A isolates (CLSI 2017). 


\section{Results and Discussion}

A total of 62 Salmonella isolates from blood samples of clinically suspected enteric fever patients were studied, of which28 isolates (45.2\%) were Salmonella typhi and 34 isolates (54.8\%) were Salmonella paratyphi A. Majority of patients were males 42 (67.7\%) with a male-to-female ratio of 2.1:1. Age of the patients ranged from 3 to 67 years, with majority of the patients (40\%) belonged to the age group of 21-30 years followed by $31-40$ years $(29 \%)$.

By disc diffusion testing, all 62 Salmonella isolates were uniformly susceptible to ampicillin, co-trimoxazole, chloramphenicol, ceftriaxone and azithromycin; whereas susceptibility to ciprofloxacin was variable and is shown in table 1.

MIC value for ciprofloxacin by E-strip test, ranged from $0.047 \mu \mathrm{g} / \mathrm{mL}$ to $>32 \mu \mathrm{g} / \mathrm{mL}$ among 62 Salmonella isolates. Highest number of isolates 15 (24.2\%) had MIC $0.5 \mu \mathrm{g} / \mathrm{mL}$, followed by $11(17.7 \%)$ isolates with MIC $0.25 \mu \mathrm{g} / \mathrm{mL}$. Six (11.5\%) isolates (5 Salmonella typhi and $1 \mathrm{~S}$. paratyphi A) had the highest MIC of $>32 \mu \mathrm{g} / \mathrm{mL}$, and totally 16 (25.8\%) isolates (7 Salmonella typhi and $9 \mathrm{~S}$. paratyphi A) had MIC in the resistance zone with MIC of $\geq 1 \mu \mathrm{g} / \mathrm{mL}$. Figure 1 shows the MIC of ciprofloxacin distribution among of
62 Salmonella isolates. Figure 2 shows $S$. typhi isolate with MIC $>32 \mu \mathrm{g} / \mathrm{mL}$.

MIC value for ceftriaxone by E-strip test, ranged from $0.012 \mu \mathrm{g} / \mathrm{mL}$ to $1.5 \mu \mathrm{g} / \mathrm{mL}$ among 62 Salmonella isolates and is shown in figure 3. Highest number of isolates $19(30.6 \%)$ had MIC of $0.25 \mu \mathrm{g} / \mathrm{mL}$, followed by $13(21 \%)$ isolates with MIC of $0.19 \mu \mathrm{g} / \mathrm{mL}$. Five (8.1\%) isolates had the MIC in the intermediate susceptible zone (1 to $1.5 \mu \mathrm{g} / \mathrm{mL}$ ), and the remaining all 57 (91.9\%) isolates were uniformly susceptible to ceftriaxone (MIC $\leq 1 \mu \mathrm{g} / \mathrm{mL})$.

Figure 4 shows Salmonella paratyphi A with MIC $1 \mu \mathrm{g} / \mathrm{mL}$ to ciprofloxacin (intermediate susceptible) and $1.5 \mu \mathrm{g} / \mathrm{mL}$ to ceftriaxone (intermediate susceptible).

MIC value for azithromycin ranged from 3 $\mu \mathrm{g} / \mathrm{mL}$ to $24 \mu \mathrm{g} / \mathrm{mL}$ among 62 Salmonella isolates and is shown in figure 5. Highest number of isolates $24(38.7 \%)$ had MIC of $12 \mu \mathrm{g} / \mathrm{mL}$, followed by $18(29.03 \%)$ isolates with MIC $8 \mu \mathrm{g} / \mathrm{mL}$. Three (4.9\%) isolates had the highest MIC in the intermediate susceptible zone $(24 \mu \mathrm{g} / \mathrm{mL})$, and the remaining $59(95.1 \%)$ isolates were uniformly susceptible to azithromycin (MIC $\leq 16 \mu \mathrm{g} / \mathrm{mL}$ ). Figure 6 shows two isolates of Salmonella paratyphi A with MIC $24 \mu \mathrm{g} / \mathrm{mL}$ to azithromycin (intermediate susceptible).

Table.1 Distribution of antimicrobial susceptibility pattern of Salmonella isolates to ciprofloxacin (both disk diffusion and MIC). Total number of isolates 62

\begin{tabular}{|l|l|l|l|l|}
\hline Ciprofloxacin & Interpretation & S.Typhi (\%) & S.Paratyphi A (\%) & Total (\%) \\
\hline \multirow{2}{*}{$\begin{array}{l}\text { Disk diffusion } \\
\text { (zone size in } \\
\mathbf{m}\end{array}$} & Sensitive & $2(3.2 \%)$ & $1(1.6 \%)$ & $3(4.8 \%)$ \\
\cline { 2 - 5 } & Intermediate & $14(22.6 \%)$ & $30(48.4 \%)$ & $44(71 \%)$ \\
\cline { 2 - 5 } & Resistant & $12(19.4 \%)$ & $3(4.8 \%)$ & $15(24.2 \%)$ \\
\hline \multirow{2}{*}{$\begin{array}{l}\text { MIC by E- } \\
\text { strip } \\
\text { (in } \boldsymbol{\mu g} / \mathbf{m L})\end{array}$} & Sensitive & $4(6.5 \%)$ & $1(1.6 \%)$ & $5(8.1 \%)$ \\
\cline { 2 - 5 } & Intermediate & $11(17.7 \%)$ & $30(48.4 \%)$ & $41(66.1 \%)$ \\
\cline { 2 - 5 } & Resistant & $13(21 \%)$ & $3(4.8 \%)$ & $16(25.8 \%)$ \\
\hline
\end{tabular}


Fig.1 MIC value of ciprofloxacin among the 62 Salmonella isolates from enteric fever cases (in $\mu \mathrm{g} / \mathrm{mL}$ )

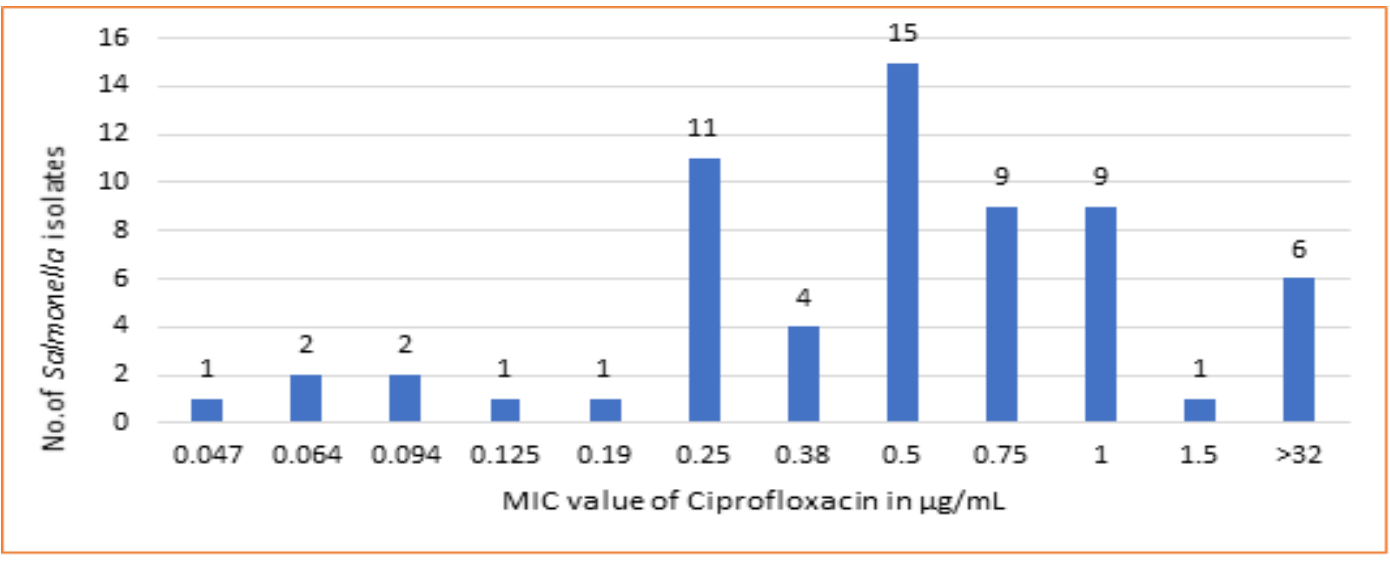

Fig.2 S. typhi isolate with MIC $>32 \mu \mathrm{g} / \mathrm{mL}$ to ciprofloxacin showing high level resistance (no inhibition ellipse seen)

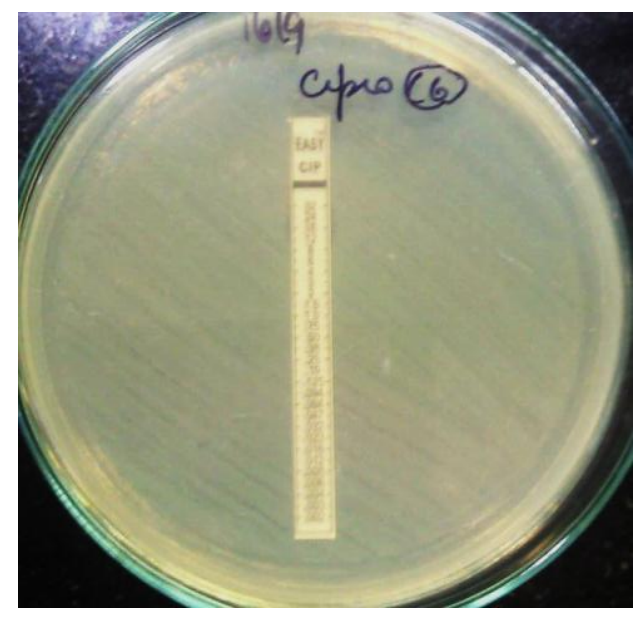

Fig.3 Distribution of MIC of ceftriaxone among the 62 Salmonella isolates

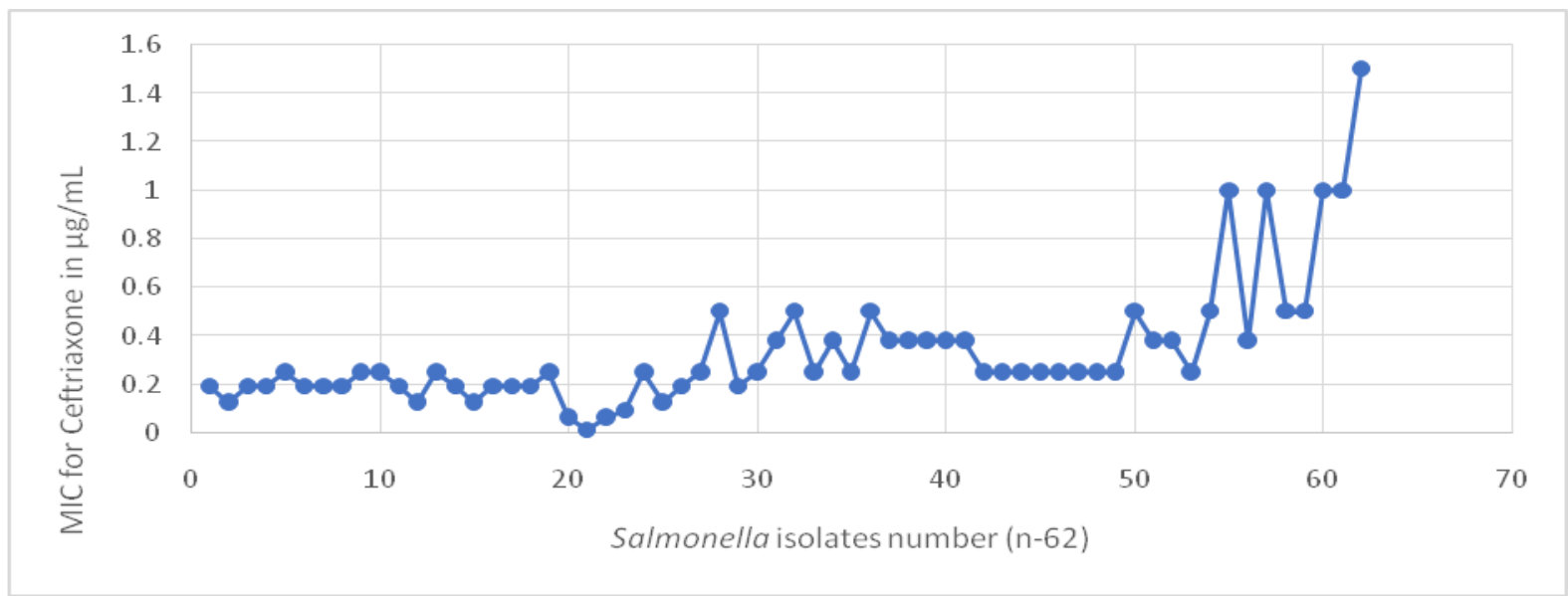


Fig.4 Salmonella paratyphi A isolate with MIC $1 \mu \mathrm{g} / \mathrm{mL}$ to ciprofloxacin (intermediate susceptible) and $1.5 \mu \mathrm{g} / \mathrm{mL}$ to ceftriaxone (intermediate susceptible)

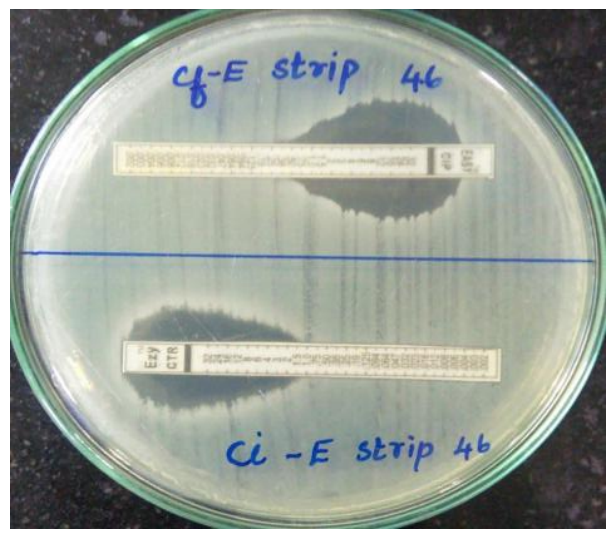

Fig.5 MIC value of azithromycin among the 62 Salmonella isolates (in $\mu \mathrm{g} / \mathrm{mL}$ )

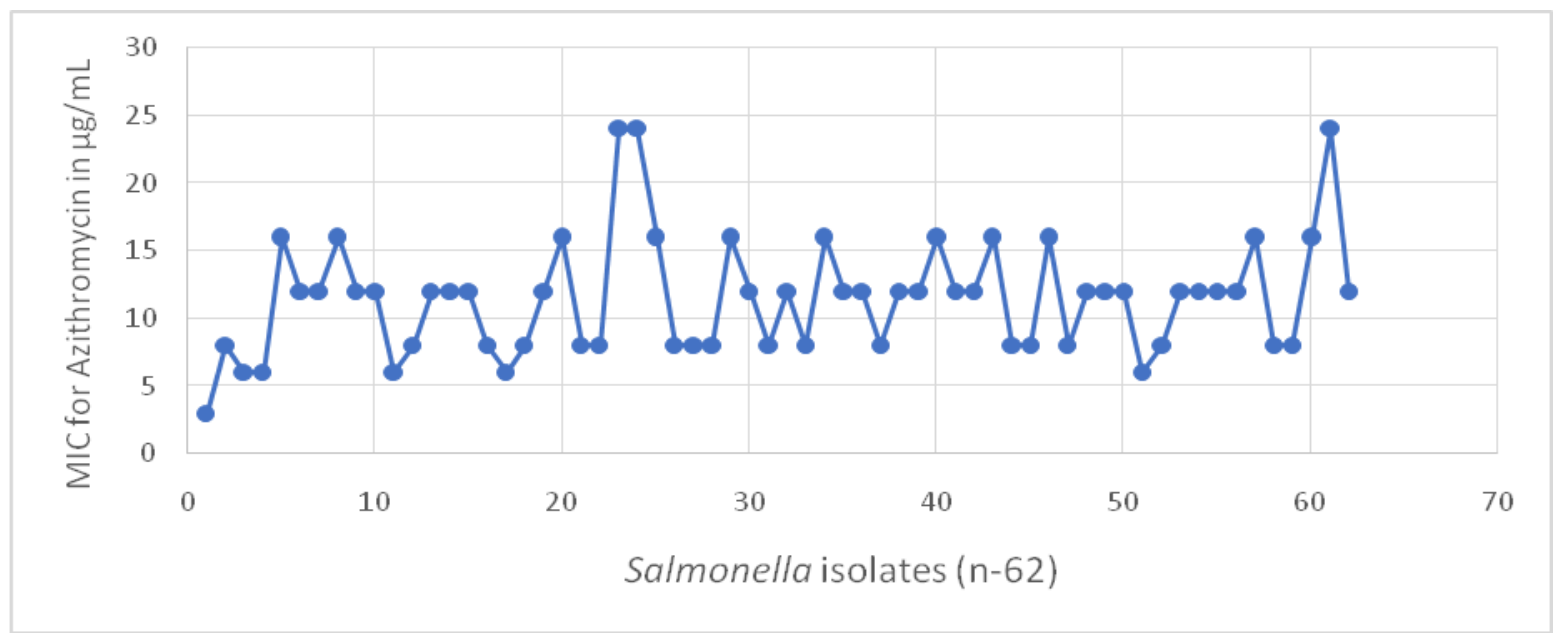

Fig.6 Two Salmonella paratyphi A isolates with MIC $24 \mu \mathrm{g} / \mathrm{mL}$ to azithromycin (intermediate susceptible)

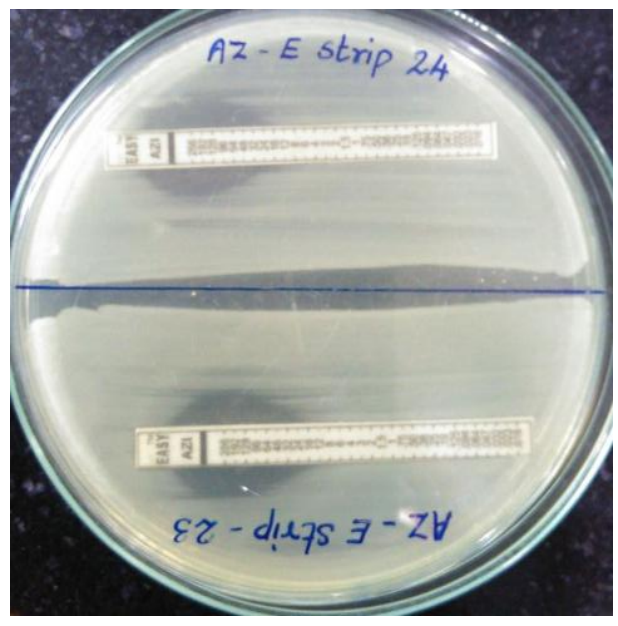


The MICs of the control strain ATTC Escherichia coli 25922 was $0.008 \mu \mathrm{g} / \mathrm{mL}$ for ciprofloxacin, $0.25 \mu \mathrm{g} / \mathrm{mL}$ for ceftriaxone, and $1.5 \mu \mathrm{g} / \mathrm{mL}$ for azithromycin.

For timely management of the enteric fever cases adequate knowledge about prevalence of the various serovars of Salmonella and their susceptibility patterns is of extreme importance. In our study, Salmonella paratyphi A outnumbered 34(54.8\%) the Salmonella typhi 28(45.2\%) isolates. Similar reports are observed in other studies across India, such as in a study from Chandigarh showed an increase of $40.6 \%$ isolation of Salmonella paratyphi A from January 2003 to April 2007 (Bharmoria and BehariVaish, 2016; Gupta et al., 2009). Recently, a multicenter surveillance study carried out by the Indian Network for Surveillance of Antimicrobial Resistance Group also showed the similar trend (Joshi, 2012). Probable reason for this observation could be explained as current vaccines offer protection against typhoid fever only. So Salmonella typhi infections are getting controlled and Salmonella paratyphi A is emerging (Bharmoria and BehariVaish, 2016).

Antimicrobial susceptibility reports serve as a guide to clinicians for selecting most appropriate therapeutic agent. In the present study, all 62 isolates were uniformly susceptible to ampicillin, chloramphenicol, co-trimoxazole, azithromycin and ceftriaxone by disc diffusion testing; whereas 15 (24.2\%) isolates were resistant and $44(71 \%)$ isolates were intermediate susceptible to ciprofloxacin by disk diffusion testing and 16 (25.8\%) isolates were resistant and $41(66.1 \%)$ isolates were intermediate susceptible to ciprofloxacin by MIC method. No isolate exhibited multidrug resistance.

Increased use of second line anti-salmonellae drugs and the discontinuation of use of the conventional first line antimicrobial agents for the treatment of the enteric fever for last two decades, probably have led to the reemergence of susceptibility to first line antibiotics. As plasmids are responsible for determining resistance to these first line antibiotics; loss of these resistance determining plasmids, may be the reason for the re-emergence of the sensitive strains (Chand et al., 2014; Shrestha et al., 2016).

In our study, discrepancy was observed for susceptibility for ciprofloxacin reports by disk diffusion and MIC method. MIC is the better predictor for drug efficacy in vivo. Also, an increase in MIC, which was not detected by disc diffusion testing is also documented by several researchers (Choudhary et al., 2013; Garg et al., 2013). Hence relying on disk diffusion test results solely may lead to delayed clinical response and serious complications (Srirangaraj, 2014).

All though no resistance was recorded to ceftriaxone or azithromycin among all 62 Salmonella isolates, we observed rising MIC to both these antimicrobial agents. Continuous monitoring of an increasing trend in MIC, is vital for early detection of emergence of drug resistance to these antibiotics (Thompson et al., 2017). According to our study results ceftriaxone and azithromycin can be still continued to be used as an effective therapeutic option for culture proven enteric fever cases.

Due to the rapid emergence widespread resistance to ciprofloxacin, prescription of the first-line drugs like ampicillin, cotrimoxazole, and chloramphenicol could be considered as treatment option for enteric fever cases in our hospital setting. This reinforces the potential need for antimicrobial recycling, wherein antibiotics that have a markedly reduced effect may be withdrawn from clinical use for a considerable period, so that they may regain 
their efficacy. However ceftriaxone and azithromycin can be still used as an effective therapeutic option for enteric fever cases in our tertiary care center; but continual monitoring of MIC and susceptibility reporting based on MIC should be routinely done, were facilities are available than doing only disk diffusion testing for enteric fever isolates to avoid treatment failures.

\section{References}

Adabara, N.U., Ezugwu, B.U., Momojimoh, A., Madzu, A., Hashiimu, Z., Damisa, D., 2012. The Prevalence and Antibiotic Susceptibility Pattern of Salmonella typhi among Patients Attending a Military Hospital in Minna, Nigeria. Adv. Prev. Med. 2012, $1-4$.

Bharmoria, A., BehariVaish, V., 2016. Analysis of Attributing Characteristics of Salmonella enteric serovar paratyphi $\mathrm{A}, \mathrm{B}$ and $\mathrm{C}$ across India during 6 years (2010 to 2015). J. Med. Microbiol. Diagn. 5. doi:10.4172/2161-0703. 1000220

Capoor, M.R., Nair, D., Deb, M., Aggarwal, P., 2007. Enteric fever perspective in India: emergence of high-level ciprofloxacin resistance and rising MIC to cephalosporins. J. Med. Microbiol. 56, 1131-32.

Chand, H.J., Rijal, K.R., Neupane, B., Sharma, V.K., Jha, B., 2014. Reemergence of susceptibility to conventional first line drugs in Salmonella isolates from enteric fever patients in Nepal. J. Infect. Dev. Ctries. 8, 1483-87.

Choudhary, A., Gopalakrishnan, R., Nambi, P.S., Ramasubramanian, V., Ghafur, K.A., Thirunarayan, M.A., others, 2013. Antimicrobial susceptibility of Salmonella enterica serovars in a tertiary care hospital in southern India. Indian J. Med. Res. 137, 800.

CLSI 2017.pdf (WWW Document), n.d. Google Docs. Performance Standards for Antimicrobial Susceptibility Testing; Twenty-Seventh Informational Supplement. CLSI document M100S27. Wayne, PA: Clinical and Laboratory Standards Institute.

Garg, A., Verma, S., Kanga, A., Singh, D., Singh, B., 2013. Antimicrobial resistance pattern and in vivo activity of azithromycin in Salmonella isolates. Indian J. Med. Microbiol. 31, 287.

Gupta, V., Kaur, J., Chander, J., others, 2009. An increase in enteric fever cases due to Salmonella paratyphi A in and around Chandigarh. Indian J. Med. Res. 129, 95.

Joshi, S., 2012. Antibiogram of S. enterica serovar typhi and $S$. enterica serovar paratyphi A: a multi-centre study from India. WHO South-East Asia J. Public Health 1, 182.

Kawser, S., Miah, M.R.A., Saleh, A.A., Sabah, K.M.N., Begum, T., 2013. Determination of minimum inhibitory concentration of Azithromycin, Ofloxacin and Ceftriaxone in Ciprofloxacin resistant Salmonella causing enteric fever. Bangladesh J. Med. Microbiol. 5, 26-30.

Koneman's Color Atlas and Textbook of Diagnostic. (WWW Document), https://shop.lww.com/Koneman-s-ColorAtlas-and-Textbook-of-DiagnosticMicrobiology/p/9781451189353. Accessed 7.7.17.

Menezes, G.A., Harish, B.N., Khan, M.A., Goessens, W.H.F., Hays, J.P., 2012. Antimicrobial resistance trends in blood culture positive Salmonella typhi isolates from Pondicherry, India, 20052009. Clin. Microbiol. Infect. 18, 239245. doi:10.1111/j.1469-0691.2011. 03546.x 
Patel, J.B., 2015. Performance standards for antimicrobial susceptibility testing. Twenty-fifth Informational Supplement. CLSI document M100- S25. Wayne, PA: Clinical and Laboratory Standards Institute.

Raveendran, R., Wattal, C., Sharma, A., Oberoi, J.K., Prasad, K.J., Datta, S., 2008. High level ciprofloxacin resistance in Salmonella enterica isolated from blood. Indian J. Med. Microbiol. 26, 50. doi:10.4103/02550857.38858

Shrestha, K.L., Pant, N.D., Bhandari, R., Khatri, S., Shrestha, B., Lekhak, B., 2016. Re-emergence of the susceptibility of the Salmonella spp. isolated from blood samples to conventional first line antibiotics. Antimicrob. Resist. Infect. Control 5. doi:10.1186/s13756-016-0121-8.
Singhal, L., Gautam, V., Gupta, P., Kale, P., Ray, P., 2014. Trends in antimicrobial susceptibility of Salmonella typhi from North India (2001-2012). Indian J. Med. Microbiol. 32, 149.

Srirangaraj, S., 2014. A study of antibiogram of Salmonella enterica serovar typhi isolates from. Australas. Med. J. 7, 185-190.

Thompson, C.N., Karkey, A., Dongol, S., Arjyal, A., Wolbers, M., Darton, T., Farrar, J.J., Thwaites, G.E., Dolecek, C., Basnyat, B., Baker, S., 2017. Treatment Response in Enteric Fever in an Era of Increasing Antimicrobial Resistance: An Individual Patient Data Analysis of 2092 Participants Enrolled into 4 Randomized, Controlled Trials in Nepal. Clin. Infect. Dis. 64, 152231.

\section{How to cite this article:}

Sandhya Bhat, K., S. Anandhalakshmi, Desdemona Rasitha, Shreyas Venkataraman and Reba Kanungo. 2017. High-Level Resistance to Ciprofloxacin and Rising MIC to Ceftriaxone and Azithromycin among Enteric Fever Isolates from a Tertiary Care Center, Puducherry, India. Int.J.Curr.Microbiol.App.Sci. 6(12): 729-736. doi: https://doi.org/10.20546/ijcmas.2017.612.076 